\title{
Bettina Greiner
}

\section{Sowjetische Speziallager in Deutschland}

\section{Anmerkungen zu einer erinnerungskulturellen "Leerstelle"}

Dass es sich bei den Speziallagern, die der sowjetische Geheimdienst NKVD mit Ende des Zweiten Weltkriegs auf dem Gebiet der sowjetischen Besatzungszone und späteren DDR errichtete, noch heute um einen „leeren“ Erinnerungsort handelt, zeigt sich nirgends deutlicher als in der Belletristik. So finden sich in der Literatur ostdeutscher Autoren allenfalls wie bei Brigitte Reimann Andeutungen auf das sowjetische Lagersystem. ${ }^{1}$ "Alles, was uns in diesen Jahren [nach 1945, B.G.] Angst gemacht hat", schreibt der Schriftsteller Günter de Bruyn über seine Themenwahl in Abhängigkeit von der Zensur, „war tabuisiert. Kein Sowjetsoldat durfte in Deutschland geplündert und vergewaltigt haben, kein nach dem Krieg Internierter in Buchenwald, Ketschendorf oder Sibirien verendet sein. " ${ }^{2}$ Doch auch in der westdeutschen Belletristik hat die stalinistische Verfolgung auf dem Gebiet der SBZ/DDR keine "Schmerzensspur“ hinterlassen, um W.G. Sebalds Diktum über die „Leerstelle“ des Luftkriegs in der Literatur aufzugreifen. ${ }^{3}$ Zwar wurde Sebalds Einschätzung umgehend widerlegt, für die Gewalterfahrung der Speziallagerhaft aber trifft sie ins Schwarze: Heinrich Cresspahl, Protagonist in Uwe Johnsons Jahrestage (1973), war für Jahrzehnte die einzige literarische Figur, deren Geschichte teilweise in einem solchen Lager spielt. ${ }^{4}$ Es dauerte mehr als 30 Jahre, bis ihm 2004 mit den beiden Berliner Schulfreunden Paul Scholz und Julian Sternberg zwei fiktive Häftlinge an die Seite gestellt wurden - in dem Jugendbuch „Julians Bruder“ von Klaus Kordon. ${ }^{5}$

Mit diesem Beispiel aus der Belletristik ist auch angezeigt, dass es in der alten Bundesrepublik keiner repressiven Geschichtspolitik wie in der DDR bedurfte, um die massiven Verfolgungserfahrungen dieser Opfergruppe unsichtbar zu machen - und dass sich an dieser Unsichtbarkeit und damit Randständigkeit des Themas innerhalb der nunmehr gesamtdeutschen Erinnerungskultur wenig geändert hat. ${ }^{6}$ Jenseits unmittelbar Betroffener und ihrer Angehörigen ist das Wissen um die Gewalt, die horrenden Todeszahlen in den Lagern und den schmalen

1 So in Brigitte Reimanns 1974 posthum in der DDR erschienenen Roman „Franziska Linkerhand“. Dort heißt es über zwei Bekannte des Vaters Linkerhand: „Der eine ist später an der Ostfront gefallen. Der andere wurde gleich nach der Kapitulation von der GPU verhaftet und starb im Lager." Brigitte Reimann: Franziska Linkerhand. Berlin 2002 (Erstersch. 1974), S. 14.

2 Günter de Bruyn: Vierzig Jahre. Frankfurt am Main 2002, S. 117.

3 W.G. Sebald: Luftkrieg und Literatur. Frankfurt am Main 2002, S. 12.

4 Uwe Johnson: Jahrestage. Frankfurt am Main 1996.

5 Klaus Kordon: Julians Bruder. Weinheim, Basel 2004.

6 Zur Wahrnehmungsgeschichte: Bettina Greiner: Verdrängter Terror. Geschichte und Wahrnehmung sowjetischer Speziallager in Deutschland. Hamburg 2010, bes. S.377-458. 
Grad zwischen Entnazifizierung, Willkür und Diktaturdurchsetzung immer noch gering. Mehr noch, es scheint, als setzte die Thematisierung eine persönliche Betroffenheit voraus. Das gilt ebenfalls für Johnson und Kordon. In Johnsons Fall verliert sich die Spur seines nach Kriegsende verhafteten und im Speziallager Fünfeichen internierten Vaters in einem Lager in der Sowjetunion; Klaus Kordon verbrachte nach einem gescheiterten Fluchtversuch längere Zeit in Haft, bevor er die DDR 1973 durch „Freikauf“ verlassen konnte. Über diesen Personenkreis hinaus scheint die Nachkriegsverfolgung in der SBZ/DDR kaum Interesse zu wecken.

Das war nicht immer so. Im frühen Kalten Krieg fanden die Überlebenden der Speziallagerhaft in Westdeutschland - besonders aber in Westberlin - breites Gehör. Dennoch sollte man die damalige politische und symbolische Wirkmächtigkeit der Opfergruppe nicht überschätzen. Dazu ist der Zusammenhang von gesellschaftlicher Wahrnehmung und politischer Instrumentalisierung vor dem Hintergrund des antikommunistischen Gründungskonsenses der Bundesrepublik zu offensichtlich. ${ }^{7}$ Vor allem aber ließ die Aufmerksamkeit prompt nach, als die DDR mit dem 17. Juni 1953 und dem Mauerbau andere politische Symbole ihres repressiven Systems „produzierte“ und die Rolle der ehemaligen Häftlinge als Kronzeugen gegen den Kommunismus damit entwertete. Fortan gab es kaum noch Anknüpfungspunkte, die ihren Erfahrungen gesamtgesellschaftliche Bedeutung hätten verleihen können. An dieser Situation hat sich mit dem Mauerfall wenig geändert. Diese Beobachtung gilt trotz beeindruckender Forschungsleistungen seit der Öffnung russischer Archive unter Präsident Jelzin. Und auch die Politik - erinnert sei an die Einsetzung der Enquete-Kommissionen des Deutschen Bundestages oder die Einberufung von Expertengremien zwecks Umgestaltung der Gedenkstätten in den neuen Bundesländern ${ }^{8}$ - blieb in dieser Hinsicht erfolglos. Trotz der Vielzahl von Gedenkorten im Osten Deutschlands und quellenfundierten wie differenzierten Ausstellungen: Es scheint, als fehle es an einem Rahmen, der historische Aufklärung und Totengedenken jenseits politischer Instrumentalisierung möglich macht.

Warum ist das so? Warum entzieht sich dieses Kapitel deutscher Gewaltgeschichte weiterhin seiner Verortung? Oder mit Blick auf die Aufmerksamkeitsökonomie gefragt: Warum bleibt es allenfalls bei kurzen Phasen medialer Aufmerksamkeit, die sich obendrein an wenigen Fingern abzählen lassen? Im Folgenden sollen drei solcher „Strohfeuer“ vorgestellt werden. Gemeint sind Situationen

7 Wolfram von Scheliha: Die sowjetischen Speziallager - ein Symbol des kommunistischen Unrechts in der publizistischen Auseinandersetzung zwischen Ost und West bis zum Bau der Mauer 1961. In: Petra Haustein, Annette Kaminsky, Volkhard Knigge, Bodo Ritscher (Hrsg.): Instrumentalisierung, Verdrängung, Aufarbeitung. Die sowjetischen Speziallager in der gesellschaftlichen Wahrnehmung 1945 bis heute. Göttingen 2006, S. 10-29; Andrew Beattie: „Sowjetische KZs auf deutschem Boden': Die sowjetischen Speziallager und der bundesdeutsche Antikommunismus“. In: Jahrbuch für historische Kommunismusforschung (2011), S. 119-137.

8 Einen konzisen Überblick bietet: Petra Haustein: Geschichte im Dissens. Die Auseinandersetzungen um die Gedenkstätte Sachsenhausen nach dem Ende der DDR. Leipzig 2006, S. 91213. 
seit dem Mauerfall, während derer die Gewalterfahrung der Speziallagerhaft vorübergehend gesellschaftliche Aufmerksamkeit erfahren hat.

\section{I.}

Das jüngste Beispiel fällt in das Frühjahr 2012: Am 18. April des Jahres wurde in Potsdam die Gedenk- und Begegnungsstätte Leistikowstraße eröffnet. Das ehemalige Untersuchungsgefängnis nahe dem Schloss Cecilienhof im Neuen Garten gehörte nicht zu den Speziallagern, die der NKVD mit Kriegsende in der SBZ installiert hatte. Es unterstand stattdessen der militärischen Spionageabwehr Smersch, die seit Einstellung der Kampfhandlungen für die operative Überwachung des sowjetischen Personals in den zahlreichen Behörden in der SBZ verantwortlich war. Dessen ungeachtet beteiligte sie sich - in ähnlicher Größenordnung wie der NKVD - an den Verhaftungen deutscher Zivilisten. ${ }^{9}$ Im Zuge weitläufiger Umstrukturierungen der Geheimdienste ging die Smersch Ende 1946 im MGB auf, sodass das Gebäude in der Leistikowstraße zunächst durch das MGB, dann das KGB genutzt wurde. Nach 1955 wurden dort ausschließlich Angehörige der Sowjetischen Armee festgehalten; Mitte der 1980er Jahre wurde das Gefängnis aufgegeben. ${ }^{10}$

Für die deutschen Gefangenen war die Leistikowstraße die erste Etappe auf ihrem Weg durch sowjetische Gefängnisse und Lager: Wer - ob als Nazi, Spion, Querulant oder wegen Schwänzens des obligaten Russischunterrichts in der Schule - in Feindverdacht geraten war, musste hier eine meist mehrmonatige Untersuchungshaft durchstehen, was bedeutete, dass die Verhafteten so lange unter „verschärften Bedingungen“ verhört wurden, bis sie die Tatvorwürfe durch eigenhändige Unterschrift der auf Russisch verfassten Vernehmungsprotokolle bestätigt hatten. Diese oft unter Folter erpressten Geständnisse stellten in der Regel das einzige Beweismittel in den anschließend inszenierten Militärtribunalen dar, die sich mehrheitlich der 14 Gummiparagrafen des Artikel 58 StGB der RSFSR von

${ }^{9}$ Vgl. Nikita Petrov: Zur Geschichte der sowjetischen Repressionsorgane (NKVD/MVD-MGB) in der SBZ 1945/46. In: Andreas Hilger, Mike Schmeitzner, Ute Schmidt (Hrsg.): Diktaturdurchsetzung. Instrumente und Methoden der kommunistischen Machtsicherung in der SBZ/DDR 1945-1955. Dresden 2001, S. 31-38, bes. S. 36.

10 Zur Geschichte des Untersuchungsgefängnisses: Elke Fein, Nina Leonhard, Jens Niederhut, Anke Höhne, Andreas Decker (Hrsg.): Von Potsdam nach Workuta. Das NKGB/MGB/KGBGefängnis Potsdam-Neuer Garten im Spiegel der Erinnerung deutscher und russische Häftlinge. Potsdam 1999; Elke Fein Nina Leonhard, Jens Niederhut: Militärstädtchen Nr. 7. Zur Geschichte des sowjetischen Untersuchungsgefängnisses Potsdam-Neuer Garten. In: Deutschland Archiv (2004) 4, S. 582-590; Peter Erler: Relikt der Unmenschlichkeit. Die sowjetische Spionageabwehr und ihr Untersuchungsgefängnis in der Potsdamer Leistikowstraße 1. In: Zeitschrift des Forschungsverbundes SED-Staat (2005) 18, S. 138-153; Von Potsdam nach Workuta. Katalog zur Ausstellung über deutsche und sowjetische Häftlinge im KBG-Gefängnis Potsdam und die Lagerhaft in Workuta/Sowjetunion. Hrsg. von Memorial Deutschland e.V., Berlin 2003; Ines Reich, Maria Schultz (Hrsg.): Sowjetisches Untersuchungsgefängnis Leistikowstraße Potsdam. Berlin 2012. 
1926 zur Ahndung konterrevolutionärer Verbrechen bedienten, um Vorwürfe wie Spionage, Sabotage, illegale Gruppenbildung und dergleichen mehr mit zehn, 15 oder 25 Jahren Lagerhaft abzustrafen. ${ }^{11}$ Nach der Verurteilung wurden die Gefangenen in eines der Speziallager in der SBZ überstellt, zur Zwangsarbeit in die Sowjetunion deportiert oder nach Moskau verbracht, wo in den ersten drei Jahren nach Wiedereinführung der Todesstrafe im Jahr 1950 mehr als 1000 Deutsche hingerichtet wurden. ${ }^{12}$

Doch nun zur Eröffnung der Gedenk- und Begegnungsstätte im April 2012, die von einem ungewöhnlichen medialen Auftrieb begleitet wurde. Einen der Gründe für diese Aufmerksamkeit erfuhren die Fernsehzuschauer von Claus Kleber, dem Moderator des „Heute Journal“ im ZDF. Er leitete den Bericht am Vorabend der Ausstellungseröffnung mit dem Hinweis auf einen prominenten Häftling ein. Dass dieser nicht in Potsdam, sondern in Schwerin unter dem Vorwurf der Spionage verhaftet, verhört und $1951 \mathrm{zu} 25$ Jahren Lagerhaft verurteilt worden war, fiel angesichts der Persönlichkeit, um die es ging, nicht ins Gewicht - sprach Klaus Kleber doch vom Vater des vier Wochen zuvor gewählten Bundespräsidenten Joachim Gauck. ${ }^{13}$ Es ist tatsächlich so banal: Prominenz verschafft Aufmerksamkeit.

In diesem Fall aber kam ein zweiter Aspekt hinzu. Etwa drei Wochen vor Ausstellungseröffnung war es $\mathrm{zu}$ einem ebenso unerfreulichen wie inakzeptablen Vorfall gekommen. Ein ehemaliger Workuta-Häftling hatte die Gedenkstättenleiterin tätlich angegriffen, weil sie ihm seines Erachtens den Zugang zum Gebäude verwehren wollte. Das Auftreten des alten Mannes ist weder kleinzureden noch zu entschuldigen. ${ }^{14}$ Es markiert jedoch den vorläufigen Höhepunkt einer jahrelangen Auseinandersetzung um die Ausstellung, die nun weit über Potsdam hinaus publik wurde - eine Auseinandersetzung zwischen der Gedenkstättenleitung auf der einen und einer großen Zahl ehemaliger Häftlinge sowie verschiedenen zivilgesellschaftlichen Organisationen, allem voran Memorial Deutschland e.V., auf der anderen Seite.

$\mathrm{Zu}$ dieser für alle Beteiligten unwürdigen Auseinandersetzung gehört folgendes Detail: Jener ehemalige Häftling war 1948 als 19-jähriger für acht Jahre nach Workuta deportiert worden, nachdem er sich - so berichtet er - einer Anwerbung

${ }^{11}$ Zur Tätigkeit der sowjetischen Militärtribunale und der Bedeutung des Art. 58: Andreas Hilger, Nikita Petrov: „Erledigung der Schmutzarbeit“? Die sowjetischen Justiz- und Sicherheitsapparate in Deutschland. In: Andreas Hilger, Mike Schmeitzner, Ute Schmidt (Hrsg.): Sowjetische Militärtribunale. Berlin 2003, Bd. 2: Die Verurteilung deutscher Zivilisten 19451955, S. 59-152; siehe auch: Friedrich-Christian Schroeder: Rechtsgrundlagen der Verfolgung deutscher Zivilisten durch sowjetische Militärtribunale. In: Ebenda, S. 37-58.

12 Erschossen in Moskau... Die deutschen Opfer des Stalinismus auf dem Moskauer Friedhof Donskoje 1950-1953. Hrsg. von Arsenij Roginskij, Jörg Rudolph, Frank Drauschke, Anne Kaminsky. Berlin ${ }^{3} 2008$.

${ }^{13} \mathrm{Zu}$ den Hintergründen der Haft: Stefan Karner: Die MGB-Akte Joachim Gauck senior. In: Frankfurter Allgemeine Zeitung vom 12.3.2012, S. 7.

14 Zwischenzeitlich wurde er zu einer Geldbuße in Höhe von $€ 1200$ verurteilt. „Eine Auffälligkeit an der Halswirbelsäule“. In: Potsdamer Neueste Nachrichten vom 31. 5. 2013, S. 7. 
als Spitzel widersetzt hatte. In einem Lager am Eismeer freundete er sich mit einem Mitgefangenen an, der die Untersuchungshaft in der Leistikowstraße hatte verbringen müssen. Diesem jüngst verstorbenen Freund zu Ehren wollte der 83-jährige nun ein öffentliches Zeitzeugengespräch organisieren. Mit diesem Herzenswunsch wandte er sich an die brandenburgische Staatskanzlei, die ihm, wie es in den „Potsdamer Neuesten Nachrichten“ heißt, schriftlich bestätigte, dass die Gedenkstättenleiterin „gebeten [wurde, solcherart] Anfragen positiv zu entscheiden ". ${ }^{15}$ So aber konnte der Eindruck entstehen, dass Zeitzeugengespräche in der Gedenk- und Begegnungsstätte allenfalls durch politische Intervention möglich sind. Schlimmer noch, dass diese Zeitzeugen stören.

Dergleichen war sicher nicht intendiert. Umso weniger, als dass sich die Gedenk- und Begegnungsstätte Leistikowstraße als „moderne Gedenkstätte“ verstanden wissen will. ${ }^{16}$ Gleichwohl irritieren Selbstdarstellung und Politik des Hauses. So hat es offenbar langer interner Auseinandersetzungen bedurft, ehe das Wort Folter in der Darstellung dieses Ortes seines angemessenen Platz fand. ${ }^{17}$ Problematisch ist jedoch nicht allein die Sprache; Fachkritik gibt es ebenfalls an handwerklichen und inhaltlichen Mängeln. ${ }^{18}$ Selbstredend sind auch positive Stimmen zu vernehmen. Martin Sabrow etwa machte jüngst darauf aufmerksam, dass die Dauerausstellung unter Historikern und Ausstellungsmachern „verbreitete Anerkennung “ finde ${ }^{19}$ - trotz Schwerpunktsetzungen, mit denen zentrale und für die Geschichte dieses Ortes elementare Aspekte zu „Verurteilung, Strafvollzug und Entlassung“ ausgeklammert und auf eine „Projektphase II“ verschoben werden. ${ }^{20}$ Das gilt auch, so Peter Jochen Winters, für „eine Darstellung des sowjetischen Gulag und des Schicksals der dorthin deportierten Häftlinge sowie ergänzende Informationen über den Widerstand in der Sowjetischen Besatzungszone und der DDR und die Verhaftung von Oppositionsgruppen bis 1955“.21

Haben die Zeitzeugen also Recht? Werden ihre Erfahrungen relativiert? Selbst wenn man diesem Vorwurf nicht folgen will, verwundern insbesondere die inhaltlichen Schwerpunktsetzungen, mit denen zwei Jahrzehnte intensiver For-

15 „Tätlicher Angriff und Todesdrohung gegen Ines Reich Leistikowstraße: Streit zwischen Ex-Häftling und Gedenkstätten-Chefin eskalierte“. In: Potsdamer Neueste Nachrichten vom 27.3. 2012.

${ }^{16}$ So in: „,Spuren zum Sprechen bringen. Interview mit Ines Reich“. In: Potsdamer Neueste Nachrichten vom 26.3.2012, S. 8.

17 Die Gravamina von Memorial Deutschland e.V. liegen ebenso vor wie ein kritisches Außengutachten durch Jörg Baberowski. Zur Kritik etwa durch Herta Müller, Nobelpreisträgerin für Literatur, oder Anna Kaminski, Direktorin der Bundesstiftung zur Aufarbeitung der SEDDiktatur vgl. „Erbitterter Streit um das Gedenken“. In: Tagesspiegel vom 19.3. 2012.

18 Peter Erler: Rezension von: Ines Reich, Maria Schultz (Hrsg.), Sowjetisches Untersuchungsgefängnis Leistikowstraße Potsdam. Berlin 2012. In: sehepunkte 12 (2012) 9 [http://www. sehepunkte.de/2012/09/21757.html; 18. 9. 2012]

${ }^{19}$ Martin Sabrow: Wenn die Zeitzeugen gehen. In: Tagesspiegel vom 26. 11.2013.

${ }^{20}$ Reich, Schultz (Hrsg.), Leistikowstraße, 2012, S. 95.

${ }^{21}$ Peter Jochen Winters: Der Streit um die Leistikowstraße in Potsdam. In: Wolfgang Benz (Hrsg.): Ein Kampf um Deutungshoheit. Politik, Opferinteressen und historische Forschung. Berlin 2012, S. 37-63, S. 62. 
schung zur sowjetischen Verfolgungspraxis in Deutschland außer Acht gelassen werden. Bis zur Realisierung der nächsten Projektphase fehlen dem Gedenkstättenbesucher daher Informationen, die zum Verständnis und zur Einordnung des historischen Geschehens grundlegend sind. Das bedeutet auch, dass Informationen fehlen, die für eine aufgeklärte Annäherung an diesen Erinnerungsort und damit an die schwierige Frage nach „Deutschen als Opfern“22 von Bedeutung wären.

II.

„Welchen Platz nehmen die sowjetischen Speziallager und das SED-Unrecht insgesamt in der deutschen Erinnerungskultur ein und in welchem Verhältnis steht es zur Erinnerung an die nationalsozialistischen Konzentrationslager?“ Diese Frage Wolfram von Schelihas - vor bald zehn Jahren formuliert - hat ausweislich der Auseinandersetzung um die Präsentation in der Leistikowstraße nichts von ihrer Aktualität verloren. ${ }^{23}$ Hier aber soll sie zunächst in die erste Zeit nach dem Mauerfall zurückführen. Auf Initiative ehemaliger Speziallagerhäftlinge wurden damals an zahlreichen Orten in der DDR nahe der vormaligen Lagerstätten und Haftorte Massengräber freigelegt. Zumindest anfänglich begleiteten die Medien in Ost und West diese Grabungen intensiv. Weil sich die Berichterstattung aber alsbald auf die sogenannten Mauerschützen und insbesondere auf Mitarbeiter der Staatssicherheit konzentrierte, verebbte das Interesse so schnell wie es aufgekommen war. Und doch hatten die wenigen Monate der Nachrichtenflut genügt, eine politische Sensation zu bewirken: Seit 1945 hatte Moskau die Existenz der Speziallager abgestritten - nun, im Juli 1990, veröffentlichte das sowjetische Innenministerium den im März 1950 nach Schließung der letzten drei (von ursprünglich zehn) NKVD-Lager verfassten Abschlussbericht.

Demnach haben ein Drittel der offiziell 122671 deutschen Häftlinge die Lager nicht überlebt. ${ }^{24}$ Auch wenn diese Zahlen binnen kurzer Zeit nach oben korrigiert

22 Die Forumulierung „Deutsche als Opfer“ orientiert sich an den Titeln der Sammelbände von Bill Niven (Hrsg.): Germans as Victims. Remembering the Past in Contemporary Germany. Basingstoke 2006, und, in leichter Variation, von Lothar Kettenacker (Hrsg.): Ein Volk von Opfern? Die neue Debatte um den Bombenkrieg. Berlin 2003, die hier stellvertretend für die deutschsprachigen Veröffentlichungen über die erinnerungskulturelle Gewichtung von deutscher Täter- und Opferschaft genannt seien, die besonders zahlreich um das Jahr 2005 - und damit anlässlich des 60. Jahrestages des Kriegsendes - publiziert wurden. Die Speziallagerhaft spielte dabei keine Rolle. Vgl. Greiner, Verdrängter Terror, bes. S. 384 oder Bettina Greiner: Sowjetische Speziallager - 60 Jahre danach. Anmerkungen zu einer verstockten Debatte. In: Deutschland Archiv 43 (2010) 2, S. 289-296.

${ }^{23}$ Wolfram von Scheliha: Sackgasse Totalitarismus. Die Forderung nach einem Gedenken an die Speziallager im Zeichen der Totalitarismustheorie führt ins erinnerungspolitische Abseits. In: Deutschland Archiv 39 (2006) 2, S. 283-290, bes. S. 284.

24 Der Bericht ist abgedruckt in: Bericht über sowjetische Internierungslager in der SBZ. In: Deutschland Archiv 23 (1990) 2, S. 1804-1810. 
wurden, bleibt die Relation unverändert: Fast jeder Dritte deutsche Gefangene ist an Hunger und Krankheiten zugrunde gegangen. ${ }^{25}$ Für die stalinistisch Verfolgten galt die Publikation dieser traurigen Zahlen als offizielle Bestätigung eines berechtigten Begehrens nach gesellschaftlicher Anerkennung. Binnen kürzester Zeit nahmen sie sich selbst jedoch als „Opfer zweiter Klasse“ wahr, verwickelt in eine heftige Auseinandersetzung, die allem voran an jenen Orten entbrannt war, an denen das NKVD wie in Sachsenhausen und Buchenwald vormalige nationalsozialistischen Konzentrationslager für seine Zwecke genutzt hatte. Dort sahen sie sich mit der „Abwehr der jüngeren Generation“ und massiver „Kritik der Holocaust-Überlebenden“ "konfrontiert, ${ }^{26}$ die sich dagegen verwahrten, „das Gedenken an die KZ-Haft mit der verdienten Haft der KZ-Aufseher zu vermischen" ${ }^{27}$ Es ist der Geschichtswissenschaft relativ schnell gelungen, diese und ähnliche Vorwürfe zu entkräften. So ist längst erwiesen, dass es sich bei der überwiegenden Mehrheit der Speziallagerinsassen um das Fußvolk der nationalsozialistischen Diktatur handelte, die sogenannten „kleinen Pgs“. Dass die relative Belastung der Häftlinge geringer ausfiel als etwa in den Internierungslagern in der amerikanischen Zone, lag auch am NKVD-Befehl Nr. 00315, mit dem Stalin angewiesen hatte, Angehörige von SS, SA und „Personal von Gefängnissen und Konzentrationslagern“ nicht in die Speziallager zu überstellen, sondern als Kriegsgefangene in die Sowjetunion $\mathrm{zu}$ deportieren. ${ }^{28}$

Die Gedenkstätten haben es sich nicht zur Aufgabe gemacht, die Auseinandersetzung um die „doppelte Vergangenheit" produktiv in die Öffentlichkeit zu tragen. Dabei gibt es keine vergleichbaren Erinnerungsorte, an denen deutsche Tätergeschichte und deutsche Opfergeschichte räumlich und zeitlich so eng miteinander verknüpft sind. Wo denn sonst, möchte man fragen, soll darüber gestritten werden, wie wir als Gesellschaft diesen beiden unterschiedlichen und doch aufeinander bezogenen Verbrechenskomplexen und Opfergruppen gerecht werden wollen? Nicht minder wichtig ist die Frage, welchen Ort die Speziallager innerhalb eines zeitgemäßen Totengedenkens einnehmen können - also eines Gedenkens, das der Tatsache gerecht wird, dass „das kulturelle Gedächtnis der Deutschen in hohem Maße durch die Erfahrung und den Umgang mit dem

${ }^{25}$ Zur Zahlendiskussion: Greiner, Verdrängter Terror, S. $10 \mathrm{f}$.

${ }^{26}$ Alexander von Plato: Lebensgeschichte und Geschichte. Ein Beispiel aus der Opferkonkurrenz des Kalten Krieges. In: Kursbuch (Juni 2002), S. 149-162, Zitate S. 159; siehe auch: Ders.: „Opfer-Konkurrenten. Die Verfolgten des NS-Regimes und der sowjetischen Besatzungsmacht im Kalten Krieg und in der Entspannungspolitik“, in: Elisabeth Domansky, Harald Welzer (Hrsg.), Eine offene Geschichte. Zur kommunikativen Tradierung der nationalsozialistischen Vergangenheit, Tübingen 1999, S. 74-92.

27 „Vorwort der Herausgeber“, in: Sowjetische Speziallager in Deutschland 1945 bis 1950, hrsg. von Sergej Mironenko, Lutz Niethammer, Alexander von Plato: Berlin 1998, Bd. 1: Studien und Berichte, S.11-19, hier S. 11.

28 „Befehl des Volkskommissars für Inneres Nr. 00315 ,Zur teilweisen Abänderung des Befehls des NKVD der UdSSR Nr. 0016 vom 11. Januar 1945““. In Ebenda, Bd.2: Dokumente zur Lagerpolitik, S. 178-180, hier S. 179. 
massenhaften Tod - als massenhaftem Töten wie Getötet-werden - als einer Signatur dieses Zeitalters geprägt worden ist“ “29

Doch an den Erinnerungsorten mit „doppelter Vergangenheit“ wird über dies Fragen allenfalls wie unter einer Käseglocke diskutiert. Ausschlaggebend dafür erscheint ein normativer Kompromiss aus den frühen 1990er Jahren. Hochrangige Historikergremien sowie vom Bundestag veranstaltete Enquete-Kommissionen beteiligten sich damals an der Kontroverse über den Ort der Speziallager im historischen Erinnern. Ihre Konzepte - allem voran bei der Umgestaltung der Gedenkstätten in den neuen Bundesländern, die vor der Herausforderung standen (und stehen), sowohl NS-Opfern als auch stalinistisch Verfolgten gerecht zu werden und darüber hinaus eine Antwort auf die DDR-Gedenkkultur zu finden, die diese Orte über Jahrzehnte geprägt hat - folgen der nach dem Historiker Bernd Faulenbach benannten Faulenbachschen Formel. Darunter ist die Maxime zu verstehen,

NS-Verbrechen nicht durch den Hinweis auf das Nachkriegsunrecht zu relativieren, dieses Unrecht aber auch nicht umgekehrt angesichts der NS-Verbrechen zu bagatellisieren. ${ }^{30}$

Tatsächlich machte die Faulenbachsche Formel die Gedenkstätten in den neuen Bundesländern damals überhaupt erst handlungsfähig. Mangels praxistauglicher Weiterentwicklung aber wirkt dieser damals so wichtige Kompromiss aus heutiger Sicht wie ein Webfehler, an dem die Erinnerung fehlläuft. Denn die Debatte um den Umgang mit der stalinistischen Verfolgung dreht sich infolge dieser Setzung fast nur um „Erinnerung“ und nicht um „Geschichte“. Oder anders gesagt: Mit Blick auf die Nachkriegsverfolgung geht es primär um das wie und nicht das woran der Erinnerung.

\section{III.}

Auch wenn es sich wiederum nur um ein mediales Strohfeuer handelte, so schaffte es die Einweihung des Speziallagermuseums in der Gedenkstätte Sachsenhausen im Dezember 2001 in die „New York Times“.31 Grund dafür war die Presseerklärung des russischen Außenministeriums anlässlich der Ausstellungseröffnung: In der Präsentation würden die „Verbrechen des Faschismus und die Handlungen der sowjetischen Besatzungsmacht“, so der Wortlaut, „auf eine Stufe gestellt“ und

${ }^{29}$ Michael Geyer: Das Stigma der Gewalt und das Problem der nationalen Identität in Deutschland. In: Von der Aufgabe der Freiheit. Politische Verantwortung und bürgerliche Gesellschaft im 19. und 20. Jahrhundert. Hrsg. von Christian Jansen, Lutz Niethammer, Bernd Weißbrot. Berlin 1995, S. 673-698, hier S. 684.

${ }^{30}$ Bernd Faulenbach: Konkurrenz der Vergangenheiten? Die Aufarbeitung des SED-Systems im Kontext der Debatte über die jüngste deutsche Geschichte. In: Annegret Stephan (Hrsg.): 1945 bis 2000. Ansichten zur deutschen Geschichte, Opladen 2002, S. 17-32, hier S. 25.

31 „Ex-Death Camp Tells Story Of Nazi and Soviet Horrors“. In: New York Times vom 13.12. 2001. 
dadurch „die Untaten von Naziverbrechern“ reingewaschen. ${ }^{32}$ Ungeachtet des Umstandes, dass diese Kritik der Ausstellung nicht gerecht wird - in Moskau hatte man sehr genaue Vorstellungen davon, woran zu erinnern sei: an angeblich gerechtfertigte und rechtmäßige Straf- und Sühnemaßnahmen. Tatsächlich fanden sich in den Speziallagern neben echten Gegnern der Besatzungsmacht - und den Tausenden, die durch Folter und Verhöre erst dazu "gemacht" wurden - zahllose Nationalsozialisten aller Belastungsgrade. Und doch waren die Inhaftierungen eines sicherlich nicht: Ausdruck einer politischen Moral.

Dass die stalinistische Justiz primär politisch bestimmt und nicht auf rechtsstaatliche Prinzipien verpflichtet war, steht außer Zweifel. Es ist daher verfehlt, die Haftmaßnahmen des NKVD in einem Atemzug mit „sowjetischer Entnazifizierung " zu nennen - die Interpretation weckt Erwartungen an eine mit westlichen Internierungspraktiken vergleichbare Auseinandersetzung mit der personellen Hinterlassenschaft des NS-Regimes, mehr noch, sie deutet eine vergleichbare Rechtsförmigkeit an. Dergleichen war aber nicht gegeben. Versteht man die alliierten Nachkriegslager gar als Ausdruck der seit der Moskauer „Erklärung über Grausamkeiten" vom Oktober 1943 mehrfach wiederholten Selbstverpflichtung der Siegermächte, staatlich sanktioniertem Unrecht demokratische Rechtstaatlichkeit entgegenzusetzen, dann wird diese Diskrepanz überdeutlich. Weder in den unmittelbaren Nachkriegsjahren noch nach der Gründung der DDR hat die sowjetische Besatzungsmacht auch nur den Versuch unternommen, diesen Vorgaben gerecht zu werden. Das Sicherheitsbegehren war immer stärker.

Die Möglichkeiten und Grenzen transitiver Justiz sind nur ein Beispiel, woran erinnert werden könnte. Es würde bedeuten, sich mit Fragen an eine Besatzungsmacht auseinanderzusetzen, die Massenverhaftungen durchführte, ihre Geheimdienste auf Zivilisten hetzte und diese fern jeder rechtsstaatlichen Regularien incommunicado in Sicherheitsverwahrung nahm oder zu horrenden Lagerstrafen beziehungsweise zum Tode verurteilte. Die ehemaligen Häftlinge sind uns hier einen Schritt voraus. Sie ahnen, dass die Beschäftigung insbesondere mit diesem Themenkomplex schwierige Fragen aufwirft. Schließlich geht es um Prinzipien, die zuvor durch die Deutschen - und durchaus unter Applaus weiter Bevölkerungsteile - ebenfalls verhöhnt worden waren. Sie befürchten, dass ihre Erfahrungen vor diesem Hintergrund als gerechte Sühne angesehen und im Ergebnis bagatellisiert werden.

Die Zeitzeugen trauen uns in dieser Angelegenheit nicht, vermutlich zu Recht. Ihr Misstrauen hat auch generationelle Gründe, sehen sich die heute über 80-jährigen insbesondere durch Wissenschaft und Gedenkstätten immer wieder auf ihre Zeit als Pimpf oder in der Hitlerjugend reduziert und in moralische Mithaftung für ältere Jahrgänge genommen. Dieser kollektive Schuldvorwurf ist ihnen als kollektiver Feindverdacht aus der Haftzeit geläufig; auch vor den sowjetischen Geheimdiensten waren alle gleich schuldig. Während sie ihnen ohnmächtig ausgeliefert waren, können sie heute auf diese Vorwürfe reagieren. Wichtigstes

32 Zit. nach Haustein, Geschichte im Dissens, S. 14. 
Medium sind ihre Erinnerungsberichte, mit und in denen sie sich auf vielfältige Weise vom Nationalsozialismus zu distanzieren suchen. Es würde zu weit führen, die Erzählmotive hier im Einzelnen aufzuführen. ${ }^{33}$ Die aus anderen Kontexten deutscher Opfernarrative bekannte Strategie, die Zeit vor dem 8. Mai 1945 auszublenden, gehört zweifellos dazu. Hinzu kommen Versuche, an das in Deutschland anscheinend noch immer verfängliche Schreckbild des „Russen“ anzuknüpfen. Dass der Sowjetunion im Zweiten Weltkrieg die meisten menschlichen Verluste abverlangt wurden, wird hingegen fast immer ausgeblendet. Der Vollständigkeit halber sind die ob ihres rassistischen Untertons unerträglichen Stereotypen zu erwähnen, die in einigen Berichten aufgerufen werden.

Diese Distanzierungsversuche lassen sich kürzer fassen: Für die Betroffenen gibt der Totalitarismus sowjetischer Prägung noch heute den alleinigen Erklärungsrahmen ihrer Leidensgeschichte vor. Selbstverständlich ist diese Willkür ein unhintergehbares Faktum. Darüber aber die nationalsozialistische Vorgeschichte auszublenden und ausnahmslos alle Speziallagerhäftlinge zu unschuldigen Opfern zu erklären, bestätigt wiederum all jene, die eine Relativierung des Nationalsozialismus befürchten. Damit schließt sich der Kreis: Wieder wird über das wie und nicht das woran der Erinnerung nachgedacht.

${ }^{33}$ Ausführlich: Greiner, Verdrängter Terror, S. 406-458. 